\title{
Modelling the Effects of Eddy Current Losses on Frequency Dependent Hysteresis in Electrically Conducting Media
}

\author{
D. C. Jiles \\ Ames Laboratory, Iowa State University, Ames, Iowa 50011
}

\begin{abstract}
In recent studies it has been shown how the effects of classical eddy current losses can be used to extend the quasi-static hysteresis model to account for the frequency dependence of hysteresis in electrically conducting media. This paper deals with the extension of the hysteresis model to include anomalous or excess losses. The instantaneous power loss due to eddy currents then consists of two terms, one of which depends on $(\mathrm{dB} / \mathrm{dt})^{2}$ and the other one on $(\mathrm{dB} / \mathrm{dt})^{1.5}$. The first term is the classical power loss and the second term, due to Bertotti, is the excess power loss. It is shown how these terms are incorporated into a time dependent hysteresis model. The main achievement is that a self-consistent model of hysteresis has been developed in which the quasi-static hysteresis curves are shown to be a limiting case of the more general frequency dependent model.
\end{abstract}

\section{INTRODUCTION}

The hysteresis model equation used in this work has been described previously [1],[2]. Recently it has been shown how the frequency dependence of hysteresis can be described in "non-conducting" media, such as high frequency ferrites [3]. In the present work, the hysteresis equation is extended to describe the frequency dependence of hysteresis in electrically conducting materials. It is assumed, for the sake of simplicity, that the magnetic field penetrates the entire crosssectional area of the material uniformly. In most practical cases, the frequency of operation of electrically conducting magnetic media, such as transformer or motor laminations, is much lower than for non-conducting media, such as high frequency inductor cores. Therefore, the effects of magnetic relaxation and resonance which have been described in the previous paper [3] can be ignored.

\section{DERIVATION OF THE FREQUENCY DEPENDENT HYSTERESIS EQUATION}

The power losses are separated into hysteresis loss $\mathrm{dW}_{\mathrm{H}} / \mathrm{dt}$, classical eddy current loss $\mathrm{dW}_{\mathrm{EC}} / \mathrm{dt}$, and anomalous (or excess) loss $\mathrm{dW}_{\mathrm{A}} / \mathrm{dt}$. The classical eddy current loss is obtained by solving the Maxwell equation $\nabla \times E=-\mathrm{dB} / \mathrm{dt}$ for a given geometry, assuming that the magnetic field penetrates uniformly

Manuscript received April 1, 1994.

This work was supported by USDOE-BES, Division of Materials Sciences, under contract number W-7405-ENG-82. throughout the material. The classical eddy current instantaneous power loss per unit volume is found to be proportional to the square of the rate of change of magnetization as discussed by Chikazumi [4]. This gives

$$
\frac{d W_{E C}}{d t}=\frac{d^{2}}{2 \rho \beta}\left(\frac{d B}{d t}\right)^{2}=\frac{\mu_{0} 2 d^{2}}{2 \rho \beta}\left(\frac{d M}{d t}\right)^{2}
$$

where $\rho$ is the resistivity in $\Omega \mathrm{m}, d$ is the cross-sectional dimension in $\mathrm{m}$ (thickness for laminations, diameter for cylinders and spheres) and $\beta$ is a geometrical factor which varies from $\beta=6$ in laminations, to $\beta=16$ in cylinders and $\beta=20$ in spheres. Under the restricted condition of sinusoidal variation of B with time, at moderate and low frequencies of magnetic field excitation, such that the flux penetration is complete, the eddy current power loss per unit volume becomes equal to the well known expression [5]

$$
\frac{d W_{E C}}{d t}=\frac{\pi^{2} B^{2}{ }_{\max } d^{2} f^{2}}{\rho \beta}
$$

where $B_{\max }$ is the peak flux density in the cycle and $f$ is the frequency in $\mathrm{Hz}$. The anomalous loss results from changes in the domain configuration, and this has been treated in detail by Bertotti [6]-[8] and Fiorillo and Novikov [9]. This component of the loss can be expressed as

$$
\frac{\mathrm{dW}_{\mathrm{A}}}{\mathrm{dt}}=\left(\frac{\mathrm{GdwH}_{\mathrm{o}}}{\rho}\right)^{1 / 2}\left(\frac{\mathrm{d} \mathrm{B}}{\mathrm{d} \mathrm{t}}\right)^{3 / 2}
$$

where $G$ is a dimensionless constant of value 0.1356 , w is the width of laminations in $m, d$ the thickness in $m, \rho$ the resistivity in $\Omega \mathrm{m}$ and $\mathrm{H}_{\mathrm{o}}$ is a parameter representing the internal potential experienced by domain walls. $\mathrm{H}_{\mathrm{o}}$ has dimensions of A. $\mathrm{m}^{-1}$ and so is equivalent to a magnetic field.

Incorporating the eddy current loss mechanisms into the theory of hysteresis allows perturbations to the hysteresis curve arising from eddy current losses to be modelled. Beginning from the quasi-static hysteresis equations given previously [10],

$$
\begin{gathered}
M_{i r r}=M_{a n}-k \delta \frac{d M_{i r r}}{d H_{e}} \\
M_{r e v}=c\left(M_{a n}-M_{i r r}\right)
\end{gathered}
$$

where $\mathrm{M}_{\mathrm{irr}}$ is the irreversible component of magnetization, $\mathrm{M}_{\mathrm{an}}$ is the anhysteretic magnetization, $\mathrm{M}_{\mathrm{rev}}$ is the reversible component of magnetization, $\mathrm{H}_{\mathrm{e}}=\mathrm{H}+\alpha \mathrm{M}$, and the hysteresis parameters $\mathrm{k}, \delta, \alpha$ and $\mathrm{c}$ have been defined elsewhere [1]. Since $M=M_{\text {irr }}+M_{\text {rev }}$, it follows that

$$
\mathrm{M}=\mathrm{M}_{\mathrm{an}}-\mathrm{k} \delta(1-\mathrm{c}) \frac{\mathrm{dM}_{\mathrm{irr}}}{\mathrm{dH}_{\mathrm{e}}}
$$

and consequently the energy equation for hysteresis becomes

$$
\mu_{\mathrm{o}} \int \mathrm{M}_{\mathrm{an}} \mathrm{dH}_{\mathrm{e}}=\mu_{\mathrm{o}} \int \mathrm{MdH}_{\mathrm{e}}+\mu_{\mathrm{o}} \mathrm{k} \delta(1-\mathrm{c}) \int \mathrm{dM}_{\mathrm{irr}}
$$


where the left hand side represents energy input, and the terms on the right hand side represent change in magnetostatic energy and energy lost to pinning (hysteresis loss) respectively. Since $M_{i r r}=(1 /(1-c))\left(M-c M_{a n}\right)$, this energy equation can be replaced by

$$
\begin{array}{r}
\mu_{0} \int \mathrm{M}_{\mathrm{an}} \mathrm{dH}_{\mathrm{e}}=\mu_{0} \int \mathrm{MdH}_{\mathrm{e}}+\mu_{0} k \delta \int\left(\mathrm{dM} / \mathrm{dH}_{e}\right) \mathrm{dH}_{e} \\
-\mu_{0} k \delta c \int\left(\mathrm{dM}_{\mathrm{an}} / \mathrm{dH}_{e}\right) \mathrm{dH}_{e}
\end{array}
$$

This equation can be modified by the addition of the eddy current loss terms on the right hand side, to give

$$
\begin{aligned}
& \mu_{0} \int M_{a n} d H_{e}=\mu_{0} \int M_{d H}+\mu_{0} k \delta \int\left(\frac{d M}{d H_{e}}\right) d H_{e}-\mu_{0} k \delta c \int \frac{d_{a n}}{d H_{e}} d H_{e} \\
& +\int \frac{\mu_{0}^{2} d^{2}}{2 \rho \beta}\left(\frac{d M}{d t}\right)^{2} d t+\int\left(\frac{G d w H_{0}}{\rho}\right)^{1 / 2}\left(\frac{\mu_{0} d M}{d t}\right)^{3 / 2} d t \quad(9)
\end{aligned}
$$

The equation now needs to be converted into manageable form. Replacing (dM/dt) ${ }^{2} \mathrm{dt}$ by $(\mathrm{dM} / \mathrm{dt})(\mathrm{dM} / \mathrm{dHe}) \mathrm{dHe}$, gives

$$
\int \frac{\mu_{0}^{2} d^{2}}{2 p \beta}\left(\frac{d M}{d t}\right)^{2} d t=\frac{\mu_{0}{ }^{2} d^{2}}{2 \rho \beta} \int\left(\frac{d M}{d t}\right)\left(\frac{d M}{d H e}\right) d H_{e} .
$$

Replacing $(\mathrm{dM} / \mathrm{dt})^{3 / 2} \mathrm{dt}$ by $(\mathrm{dM} / \mathrm{dt})^{1 / 2}(\mathrm{dM} / \mathrm{dHe}) \mathrm{dHe}$ gives

$$
\begin{aligned}
& \int\left(\frac{G d w H_{0}}{\rho}\right)^{1 / 2}\left(\frac{\mu_{0} d M}{d t}\right)^{3 / 2} d t= \\
& \left(\frac{\mu_{0} G d w H_{0}}{\rho}\right)^{1 / 2} \mu_{0} \int\left(\frac{d M}{d t}\right)^{1 / 2} \frac{d M}{d H_{e}} d_{e} .
\end{aligned}
$$

Substituting this back into the hysteresis equation, (9), and dividing by $\mu_{0}$ gives

$$
\begin{aligned}
& \int M_{a n} d_{e}\left.=\int M d H_{e}\right)+k \delta \int\left(\frac{d M}{d H_{e}}\right) d H_{e}-k \delta c \int \frac{d_{a n}}{d H_{e}} d H_{e} \\
&+ \frac{\mu_{0} d^{2}}{2 \rho \beta} \int\left(\frac{d M}{d t}\right)\left(\frac{d M}{d H_{e}}\right) d H_{e} \\
&+\left(\frac{\mu_{0} G d w H_{0}}{\rho}\right)^{1 / 2} \int\left(\frac{d M}{d t}\right)^{1 / 2} \frac{d M}{d H_{e}}{d H_{e}}^{1 / 2}
\end{aligned}
$$

and differentiating this equation leads to

$$
\begin{aligned}
M_{a n}= & M+k \delta \frac{d M}{d H_{e}}-k \delta c \frac{d M_{a n}}{d H_{e}}+\frac{\mu_{o} d^{2}}{2 \rho \beta}\left(\frac{d M}{d t}\right) \frac{d M}{d H_{e}} \\
& +\left(\frac{\mu_{0} G d w H_{0}}{\rho}\right)^{1 / 2}\left(\frac{d M}{d t}\right)^{1 / 2} \frac{d M}{d H_{e}}
\end{aligned}
$$

which is equivalent in form to the quasi-static hysteresis equation, but with a modified energy loss parameter, which now includes pinning and the effects of eddy currents.

This equation can be rearranged in terms of the differential susceptibility, $\mathrm{dM} / \mathrm{dH}$, to give

$$
\begin{gathered}
\left(\frac{\mu_{0} d^{2}}{2 \rho \beta} \frac{d H}{d t}\right)\left(\frac{d M}{d H}\right)^{2}+\left(\frac{\mu_{0} G d w H_{0}}{\rho}\right)^{1 / 2}\left(\frac{d H}{d t}\right)^{1 / 2}\left(\frac{d M}{d H}\right)^{3 / 2} \\
+\left(k \delta-\alpha\left(M_{a n}-M+k \delta c \frac{d M_{a n}}{d H_{e}}\right)\right)\left(\frac{d M}{d H}\right) \\
-\left(M_{a n}-M+k \delta c \frac{d M_{a n}}{d H_{e}}\right)=0
\end{gathered}
$$

The equation was then solved for $(\mathrm{dM} / \mathrm{dH})$ numerically using the Newton-Raphson method.

\section{RESULTS}

In order to test the predictions of the model, comparisons were made with published data for permalloy [11]. The hysteresis curves of permalloy at different frequencies are shown in Fig. 1. The main points to note are that the coercivity increases with frequency (from $2.4 \mathrm{~A} \cdot \mathrm{m}^{-1}$ at dc, to $5.9 \mathrm{~A} \cdot \mathrm{m}^{-1}$ at $6 \mathrm{kHz}$ ), while the remanence is invariant with frequency. The behavior after reversal at the hysteresis loop tips results in an immediate reduction in magnetization, so that there is no rounding of the loop tips. The modelled hysteresis curves are shown in Figs. 2 and 3. The values of the parameters used were $B_{s}=1$ Tesla, $a=3.75 \mathrm{~A}^{-1} \mathrm{~m}^{-1}, \mathrm{k}=2.4 \mathrm{~A} \cdot \mathrm{m}^{-1}, \alpha=1.5 \mathrm{x}$ $10^{-5}$, and $c=0.35$ (which define the quasi static hysteresis loop, and have been discussed previously [1]), and $\rho=0.57 \mathrm{x}$ $10^{-6} \Omega . \mathrm{m}, \mathrm{d}=1.5 \times 10^{-5} \mathrm{~m}, \beta=6, \mathrm{w}=0.005 \mathrm{~m}$ (which define the eddy current losses). For classical loss only $\mathrm{H}_{0}=0$ A. $\mathrm{m}^{-1}$ and the hysteresis curves are shown in Fig. 2, while for classical plus anomalous loss $\mathrm{H}_{0}=0.0075$ A.mresulting in the hysteresis curves shown in Fig. 3. It is noticeable that the model correctly predicts the invariance of remanence with frequency and also the persistence of sharp loop tips up to $6 \mathrm{kHz}$. A summary of the resulting modelled hysteresis curve data is given in Tables I and II. It can be seen that there is good agreement between the measured and modelled data under quasi-static conditions in Table $\mathrm{I}$. The variation of coercivity with frequency is shown in Table II.

\section{CONCLUSIONS}

The work presented here shows how the quasi-static hysteresis equation can be extended to account for energy losses resulting from the generation of eddy currents in electrically conducting media. The model represents a first approximation in which the skin effect is ignored, or equivalently the field penetration is assumed to be uniform throughout the material. This means that the calculations are really only applicable to thin laminations. The model uses the standard quasi-static hysteresis loss with the addition of two extra terms representing the classical eddy current power loss and the anomalous (or excess) eddy current power loss.

The results show the increase in coercivity with frequency, the invariance of the remanence with frequency, the increase in energy loss per cycle and the persistence of sharp reversal points at the loop tips. The model provides a relatively simple way of predicting the changes in hysteresis curves as a result of eddy currents at different frequencies. The information needed is limited to the quasi-static hysteresis curve of the material, the resistivity, and the shape of the component (e.g., lamination, cylinder), including its cross-sectional thickness (e.g., lamination thickness or cylinder diameter), the width and the internal potential as represented by the field $\mathrm{H}_{0}$.

Future work should include a means of correcting these curves for the effects of depth of penetration in conducting media. This would enable the model to be extended to "thick" 
components in which the energy dissipation at a given frequency was not uniform over the entire cross-section.

\section{ACKNOWLEDGMENT}

The author thanks Dr. G. Bertotti for useful discussions.

\section{REFERENCES}

[1] D. C. Jiles and D. L. Atherton, J. Mag. Mag. Mater. vol. 61, p. 48 1986.

[2] D. C. Jiles and J. B. Thoelke, IEEE Trans. Mag., vol. 25, p. 3928, 1989.

[3] D. C. Jiles, IEEE Trans. Mag., vol. 29, p. 3490, 1993

[4] S. Chikazumi, Physics of Magnetism. New York: John Wiley, 1964 p. 321 .

[5] D. C. Jiles, Introduction to Magnetism and Magnetic Materials London: Chapman \& Hall, 1991, p. 271.

[6] G. Bertotti, J. Appl, Phys., vol. 55, pp. 4339, 1984

7] G. Bertotti, J. Appl. Phys, vol. 57, pp. 2110, 1985.

[8] G. Bertotti, IEEE Trans. Mag, vol. 24, pp. 621, 1988

[9] F. Fiorillo and A. Novikov, IEEE Trans. Mag., vol. 26, pp. 2559 1990.

[10] D. C. Jiles, J. B. Thoelke and M. K. Devine, IEEE Trans. Mag., vol 28 , pp. 27-35, 1992.

[11] "Design manual for tape wound cores," Magnetics Inc., Components Division, 1987, p. 37

TABLE I.

Comparison of Measured and Modelled Magnettc Propertites of Square

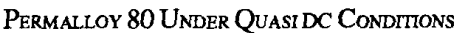

\begin{tabular}{lll}
\hline \hline & (Measured) & (Modelled) \\
\hline Saturation magnetic induction & $1.05 \mathrm{Tesla}$ & $1.0 \mathrm{Tesla}$ \\
Anhysteretic permeability & $-1.16 \times 10^{6}$ \\
Initial permeability & $26 \times 10^{3}$ & $25.5 \times 10^{3}$ \\
Field at loop tip & $10 \mathrm{~A} / \mathrm{m}$ & $10 \mathrm{~A} / \mathrm{m}$ \\
Magnetic induction at loop tip & $0.76 \mathrm{Tesla}$ & $0.78 \mathrm{Tesla}$ \\
Differential permeability at loop tip & $12.5 \times 10^{3}$ & $13.3 \times 10^{3}$ \\
Magnetic induction at remanence & $0.62 \mathrm{Tesla}$ & $0.59 \mathrm{Tesla}$ \\
Differential permeability at remanence & $27.5 \times 10^{3}$ & $52 \times 10^{3}$ \\
Coercivity & $2.4 \mathrm{~A} / \mathrm{m}$ & $2.5 \mathrm{~A} / \mathrm{m}$ \\
Differential permeability at coercive point & $1.0 \times 10^{6}$ & $1.7 \times 10^{6}$ \\
\hline
\end{tabular}

TABLE II

Comparison of Mrasurpd and Model Led Values of Coerctivty of SQuare Permalloy 80 at Different Frequencies

\begin{tabular}{|c|c|c|c|}
\hline \multirow[t]{2}{*}{ Frequency $(\mathrm{Hz})$} & \multirow[t]{2}{*}{$\begin{array}{l}\mathrm{H}_{\mathrm{c}}\left(\mathrm{A}^{-1}\right) \\
\text { (measured) }\end{array}$} & \multicolumn{2}{|c|}{$\begin{array}{l}\mathrm{H}_{\mathrm{c}}\left(\mathrm{A} \cdot \mathrm{m}^{-1}\right) \\
\text { (modelled) }\end{array}$} \\
\hline & & $\begin{array}{l}\text { classical } \\
\text { loss only }\end{array}$ & $\begin{array}{l}\text { classical }+ \\
\text { excess losses }\end{array}$ \\
\hline $\begin{array}{r}\text { "DC" (5) } \\
400 \\
1000 \\
3000 \\
6000\end{array}$ & $\begin{array}{l}2.4 \\
2.9 \\
3.6 \\
4.6 \\
5.9\end{array}$ & $\begin{array}{l}2.4 \\
2.8 \\
3.1 \\
3.7 \\
4.4\end{array}$ & $\begin{array}{l}2.5 \\
3.5 \\
4.0 \\
4.9 \\
5.6\end{array}$ \\
\hline
\end{tabular}

These values were obtained using the following model parameters: $B_{s}=1$

Tesla, a $=3.75 \mathrm{~A} . \mathrm{m}^{-1}, \mathrm{k}=2.4 \mathrm{~A} \cdot \mathrm{m}^{-1}, \alpha=1.5 \times 10^{-5}, \mathrm{c}=0.35, \rho=0.57 \mathrm{x}$

$10^{-6} \Omega \mathrm{m}, \mathrm{d}=1.5 \times 10^{-5} \mathrm{~m}, \beta=6, \mathrm{w}=0.005 \mathrm{~m}, \mathrm{H}_{\mathrm{o}}-0.0075{\mathrm{~A} . \mathrm{m}^{-1}}^{-1}$

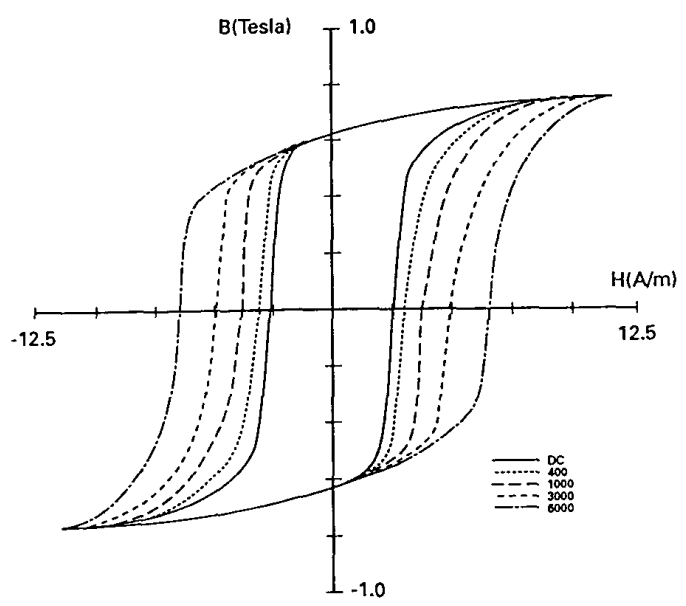

FIG. 1. Hysteresis curves of permalloy 80 at $0,400,1000,3000$ and 6000 $\mathrm{Hz}$ [11].

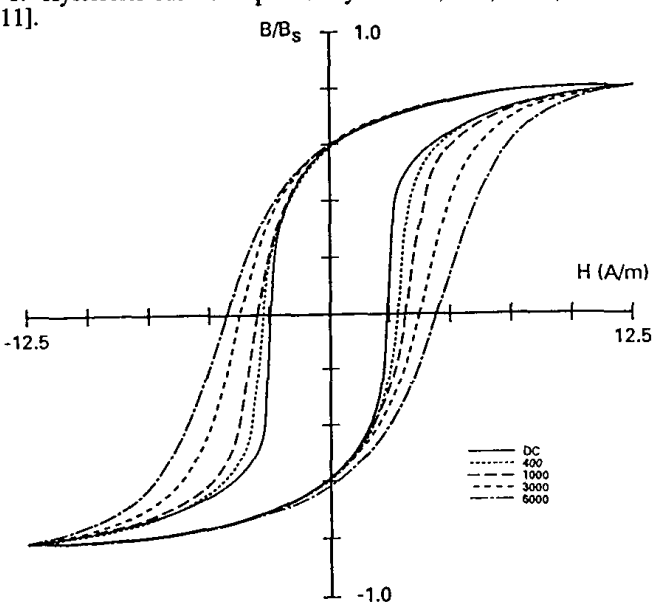

FIG. 2. Modelled hysteresis curves of permalloy 80 at $0,400,1000,3000$ and $6000 \mathrm{~Hz}$, including the classical eddy current loss only $\left(\mathrm{H}_{\mathrm{o}}=0 \mathrm{~A} \cdot \mathrm{m}^{-1}\right)$.

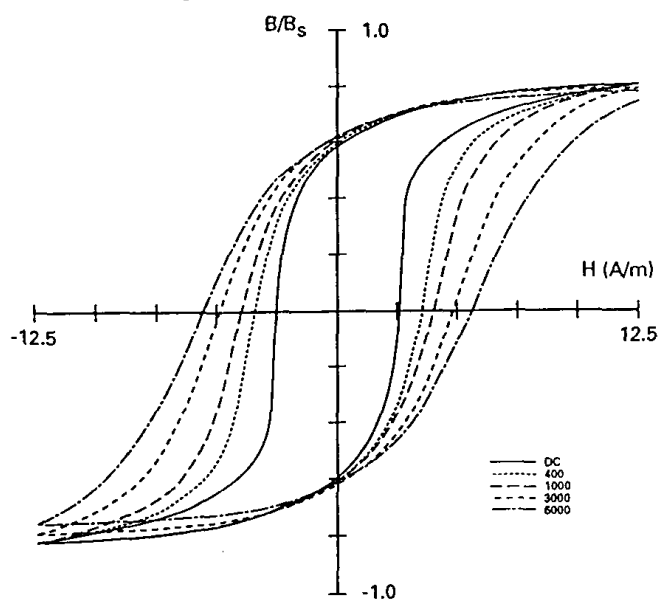

FIG. 3. Modelled hysteresis curves of permalloy 80 at $0,400,1000,3000$ and $6000 \mathrm{~Hz}$, including both classical and anomalous eddy current losses $\left(\mathrm{H}_{\mathrm{o}}=0.0075\right.$ A.m $\left.{ }^{-1}\right)$ 